\title{
Diabetes Mellitus pós-transplante (DMPT)
}

Isabelly de Oliveira Pinheiro ${ }^{1}$. Daniel Duarte Gadelha². Manuela Montenegro Dias de Carvalho². Paulo Cruz de Queiroz ${ }^{2}$. Virginia Oliveira Fernandes ${ }^{3}$. Renan Magalhães Montenegro Junior ${ }^{4}$.

1 Residente em Endocrinologia, Hospital Universitário Walter Cantídio (HUWC), Fortaleza, Ceará, Brasil. 2 Endocrinologista, Hospital Universitário Walter Cantídio (HUWC), Fortaleza, Ceará, Brasil. 3 Doutorado em Ciências Médicas, Universidade Federal do Ceará (UFC), Fortaleza, Ceará, Brasil. 4 Doutorado em Clínica Médica, Gerente de Ensino dos Hospitais Universitários da Universidade Federal do Ceará (UFC), Fortaleza, Ceará, Brasil.

\section{Importância}

- O Diabetes Mellitus pós-transplante (DMPT) ocorre em 10\% a 40\% dos pacientes que receberam transplante de órgãos sólidos;

- Essa incidência é variável e depende dos critérios utilizados para o seu diagnóstico, da população avaliada, do tipo de transplante, dos fatores de risco pré-transplante e dos medicamentos imunossupressores utilizados;

- É de fundamental importância entender o papel das drogas imunossupressoras, seu potencial diabetogênico e suas interações medicamentosas, além de compreender como é feito o diagnóstico de DMPT e seu adequado tratamento.

\section{Avaliação pré-transplante}

- Todo paciente candidato a transplante de órgão sólido deve ser avaliado quanto a ocorrência de Diabetes Mellitus tipo 2 (DM2) prévio não diagnosticado e quanto a fatores de risco comuns ao DM2 e DMPT:

- Idade > 40 anos;

- Síndrome metabólica;

○ Obesidade;

○ Intolerância à glicose.

DMPT: como rastrear?

- Realizar dosagem de glicemia de jejum e/ou TOTG (75 g) no pós-transplante:

- Semanalmente no primeiro mês;

- A cada 3 meses durante o primeiro ano.

- Após, anualmente.

\section{DMPT: como diagnosticar?}

- Os critérios diagnósticos são os mesmos utilizados para diagnóstico de Diabetes Mellitus na população geral (Sociedade Brasileira de Diabetes, 2018; American Diabetes Association, 2019):

- Glicemia de jejum $\geq 126 \mathrm{mg} / \mathrm{dL}$ (teste repetido e confirmado);

- Glicemia plasmática $\geq 200 \mathrm{mg} / \mathrm{dL}$ associado a sintomas clássicos de hiperglicemia;

- Glicemia $2 \mathrm{~h}$ após $75 \mathrm{~g}$ de glicose $\geq 200 \mathrm{mg} / \mathrm{dL}$;

- $\mathrm{HBA} 1 \mathrm{C} \geq 6,5 \%$ (teste repetido e confirmado).

- O diagnóstico de DMPT necessariamente precisa ser feito após alta hospitalar, quando o paciente estiver com dose estável de droga imunossupressora.

\section{A Glicohemoglobina (A1c) é confiável no DMPT?}

- A A1c como método isolado NÃO é confiável no primeiro ano após transplante;

- Fatores no pós-transplante que podem interferir na A1c são: anemia, transfusão sanguínea, uremia, terapêutica com eritropoietina, além da redução do tempo de sobrevida das hemácias após o transplante. 


\section{Qual o papel das drogas imunossupressoras no desenvolvimento do DMPT?}

Mecanismos de ação diversos podem estar implicados, porém uma determinada droga apresenta predominância de um deles, como se segue na tabela abaixo:

\begin{tabular}{|l|l|}
\hline \multicolumn{1}{|c|}{ Droga } & \multicolumn{1}{|c|}{ Mecanismo da hiperglicemia } \\
\hline Tacrolimus / ciclosporina & Redução da secreção de insulina \\
\hline Glicocorticóides & $\begin{array}{l}\text { Resistência periférica à insulina } \\
\text { Aumento da produção hepática de glicose }\end{array}$ \\
\hline Sirolimus / Everolimus & $\begin{array}{l}\text { Redução do sinal de transdução da insulina } \\
\text { Indução de apoptose de células- } \beta\end{array}$ \\
\hline Micofenolato mofetil / azatioprina & Sem efeitos proeminentes \\
\hline
\end{tabular}

\section{DMPT: como tratar?}

- Durante os primeiros dois meses após o transplante, a insulinoterapia é preferível;

- Considerar a retirada de insulina em favor da terapia oral quando a necessidade de insulina for $<20$ unidades por dia;

- Vários medicamentos orais são validados para tratamento, sendo os mais recomendados: metformina, pioglitazona e inibidores da DPP-4. A escolha depende do perfil do paciente e de seus efeitos colaterais;

- Não há estudos com análogos do GLP-1 e inibidores de SGLT-2 que demonstrem claro benefício e segurança na DMPT, até este momento.

\section{Hiperglicemia no pós-transplante: o que fazer?}

- Aproximadamente 90\% dos receptores de órgãos sólidos exibem hiperglicemia nas primeiras semanas após o transplante;

- Essa hiperglicemia é induzida por estresse cirúrgico e/ou devido às altas doses de corticosteroides utilizadas como terapia de indução imunossupressora;

- É recomendado o tratamento intensivo da hiperglicemia durante internamento com insulinoterapia, visto que a hiperglicemia persistente após o transplante é responsável pelo risco aumentado de evolução para DMPT, devido a glicotoxicidade;

- Na maioria dos casos, a hiperglicemia se resolve antes da alta.

\section{REFERÊNCIAS}

1 Jenssen T, Hartmann A. Post-transplant diabetes mellitus in patients with solid organ transplants. Nat Rev Endocrinol. 2019;15(3):172-88.

2 Shivaswamy V, Boerner B, Larsen J. Post-transplant diabetes mellitus: causes, treatment, and impact on outcomes. Endocr Rev. 2016;37(1):37-61.

3 Sharif A, Cohney S. Post-transplantation diabetes - state of the art. Lancet Diabetes Endocrinol. 2016;4(4):337-49.

4 Ferreira R, Oliveira M, Vasconcelos C. Diabetes mellitus pós-transplante renal: new-onset diabetes after kidney transplantation. Revista Portuguesa de Diabetes. 2015;10(4):151-7.

5 Davidson JA, Wilkinson A; International Expert Panel on New-Onset Diabetes after Transplantation. New-onset diabetes after transplantation 2003 International Consensus Guidelines: an endocrinologist's view. Diabetes Care. 2004;27(3):805-12.

6 Gupta S, Pollack T, Fulkerson C, Schmidt K, Oakes DJ, Molitch ME, et al. Hyperglycemia in the posttransplant period: NODAT vs Posttransplant Diabetes Mellitus. J Endocr Soc. 2018;2(11):1314-19.

7 Oliveira EP, Montenegro RM Junior, Vencio S, organizadores. Diretrizes da Sociedade Brasileira de Diabetes 2017-2018. São Paulo: Editora Clannad; 2017 [acesso em: 25 mar 2019]. Disponível em: http://www.diabetes.org.br/profissionais/images/2017/diretrizes/diretrizessbd-2017-2018.pdf

8 American Diabetes Association. Classification and diagnosis of diabetes: standards of medical care in diabetes - 2019. Diabetes Care. 2019;42(Supl. 1):S13-28.

\section{Como citar:}

Pinheiro IO, Gadelha DD, Carvalho MM, Queiroz PC, Fernandes VO, Montenegro RM Junior. Diabetes Mellitus Pós-transplante (DMPT). Rev Med UFC. 2019 jan-mar;59(1):77-78. 